\title{
Advances in applied computational intelligence: MICAI 2016
}

\author{
Hiram Ponce ${ }^{1} \cdot$ Miguel González-Mendoza $^{2} \cdot$ Lourdes Martínez-Villaseñor $^{1}$
}

This special issue of the Journal Computing offers original contributions in all areas of artificial intelligence. Most of the research works included in this issue are extended papers presented in the 15th Mexican International Conference on Artificial Intelligence, MICAI 2016, held in Cancún, Quintana Roo, Mexico on October 23-29, 2016, under the organization of the Mexican Society for Artificial Intelligence (SMIA) in cooperation with the Instituto Tecnológico de Cancún. Other papers included were received through the open call for papers.

MICAI is an annual conference that disseminates and promotes growth of outstanding research works in all areas of artificial intelligence (AI), including but not limited to: expert systems and knowledge-based systems, knowledge representation and acquisition, multi-agent systems and distributed AI, natural language processing, intelligent interfaces, computer vision, machine learning, pattern recognition, soft computing, reasoning, robotics, planning and scheduling, among others.

In MICAI 2016, we received over 350 submissions with an acceptance rate around $25 \%$. Based on the reviewers' comments from the conference, 15 papers were initially invited to submit to the special issue after substantial extension. Additionally, three manuscripts from the open call for papers were received. Each submission was

Hiram Ponce

hponce@up.edu.mx

Miguel González-Mendoza

mgonza@itesm.mx

Lourdes Martínez-Villaseñor

lmartine@up.edu.mx

1 Facultad de Ingeniería, Universidad Panamericana, Campus México, Augusto Rodin 498, 03920 Mexico, Ciudad de México, Mexico

2 Tecnológico de Monterrey, Escuela de Ingeniería y Ciencias, 52179 Atizapan de Zaragoza, Estado de México, Mexico 
reviewed by at least two reviewers in order to guarantee a successful peer-review process expecting originality, quality, correctness and relevance.

We selected five papers that cover different areas of AI with special emphasis in the integration of theory and applications. The topics of these works consider natural language processing, machine learning, knowledge information, reasoning, and creativity.

An interesting method of computational creativity is presented by López-Ortega et al. in the paper An intelligent multi-agent system to create and classify fractal music. The authors developed a multi-agent system to create fractal music seeking to provoke an emotional response in the listener. Their proposed system also classifies fractal music pieces according to emotional labels taken from the Circumplex Model of Affect (CMoA) proposed by Russell. The method of music creation is based on information fusion and discover of knowledge is done using an ensembles of clusters.

A noteworthy effort to query uncertain XML data is proposed by Ma et al. in the paper *An approach of top-k keyword querying for fuzzy $X M L^{*}$. Their approach for top- $k$ keyword queries over fuzzy XML documents use fuzzy SLCA semantics for the queries, and a novel encoding based on Dewey. The new algorithms Fstack and Mindex to find top- $k$ SLCA results and their possibilities proved to be efficient and effective.

Big data for knowledge-discovery in agro-climatic applications is proposed by Mohammed, et al. in the paper Fuzzy based approach for discovering crops plantation knowledge from huge agro-climate data representing climate changes. Their approach consists on a fuzzy system that extracts information from a huge agro-climate data to determine crop plantation dates, harvesting dates, expected diseases and water requirements in crops. The application was validated in a large database from Egypt showing effectiveness in comparison to other works.

The theoretical paper entitled Inverse formulas of parameterized orthogonal wavelets by Herrera-Alcántara, et al. presents a study of the parameterization of orthogonal wavelet-based filters and their inverse formulas. They suggest image processing as an application of the optimized formulation of the parameters of these filters to maximize the number of negligible wavelet coefficients.

Lastly, Gómez-Adorno, et al. in the paper Document embeddings learned on various types of $\mathrm{n}$-grams for cross-topic authorship attribution proposes to represent the components vector of a text using a modified version of the Paragraph Vector method (Doc2vec). In this work, the authors explore to use the document embeddings learned by Doc2vec using not only words, but also $n$-grams of characters, words and POS tags. They claimed that it models the semantics of documents along with their syntaxis. Also, they demonstrated that this approach improves the performance of cross-topic authorship attribution.

We thank all the authors for their contributions as well as all the reviewers for their valuable comments. We also thank the editorial team of the journal for their support in the publication of this special issue.

\section{Compliance with ethical standards}

Conflict of interest The authors declare that they have no conflict of interest. 
*Note added in proof: The above mentioned article by Zongmin Ma, Ting Li, Li Yan: An approach of top-k keyword querying for fuzzy XML, was planned to be part of this Special Issue on MICAI 2016 but was mistakenly published in vol. 100, issue 3 (2018): Ma, Z., Li, T. \& Yan, L.: An approach of top-k keyword querying for fuzzy XML*. Computing (2018) 100: 303-330. The online version of the original article can be found at https://doi.org/10.1007/s00607-017-0577-2. 